\title{
CAISSE NATIONALE SUISSE D'ASSURANCE EN CAS \\ D'ACCIDENTS: RÉSULTATS DE LA STATISTIQUE DES ACCIDENTS DE LA HUITIĖME PÉRIODE \\ QUINQUENNALE I953-I957
}

HaNS KoCH,

Lucerne

La Caisse nationale suisse d'assurance en cas d'accidents à Lucerne, chargée du service de l'assurance obligatoire en cas d'accidents en Suisse, a publié il y a quelque temps les „Résultats de la statistique des accidents de la huitième période quinquennale I953-I957". Cette publication, qui est la huitième, paraît tous les cinq ans en français et en allemand *). Ces différents rapports donnent un aperçu intéressant de la situation de l'assurance-accidents obligatoire en Suisse. Ils constituent dans leur ensemble une documentation précieuse touchant l'un des aspects du développement de la sécurité sociale en Suisse depuis I9I8. C'est en effet à cette date que l'assurance obligatoire en cas d'accidents est entrée en vigueur et que la Caisse nationale suisse d'assurance en cas d'accidents, établissement de droit public autonome, a commencé son activité.

Le dernier rapport sera aussi consulté avec fruit par quiconque s'intéresse aux problèmes de l'assurance sociale. Cette publication d'environ I 80 pages, illustrée par de nombreux tableaux, graphiques et illustrations, fournit une quantité d'indications très utiles présentées de façon claire. Les premiers chapitres sont surtout consacrés aux statistiques tirées du règlement des sinistres, tandis que les derniers chapitres traitent de quelques problèmes d'assurance spéciaux qui sont particulièrement actuels.

Le chapitre intitulé Effectif assuré étudie les grandeurs des différents effectifs en faisant allusion aux problèmes posés par leur

*) Ergebnisse der Unfallstatistik der achten Beobachtungsperiode r9531957 . 
détermination. Les deux chapitres suivants traitent du Nombre des accidents et du Cồt des accidents. On y aborde aussi plusieurs questions intéressantes, par exemple les difficultés d'enregistrement dues à la délimitation des différentes catégories d'accidents ou les causes des divergences relevées dans l'évolution nominale des composantes du coût des accidents. Les éléments de base (effectif assuré, nombre et coût des accidents), établis dans les trois premiers chapitres, sont ensuite comparés les uns aux autres dans un quatrième chapitre et fournissent les données nécessaires à la mesure de la Fréquence et de la gravité des accidents. On y soulève entre autres la question de la comparabilité des grandeurs du risque-accidents sur le plan international. Le chapitre suivant traite des statistiques très étendues que nécessite le contrôle des valeurs actuelles tirées des propres expériences de la Caisse nationale, qui doit appliquer le système des réserves mathématiques pour les rentes. Ce chapitre intitulé Rentes d'invalidité et de survivants présente un intérêt général au point de vue actuariel.

Un des problèmes spéciaux traités dans ce rapport est relatif à la prévention des accidents. Les enquêtes menées sur les Causes d'accidents dans les fonderies et dans l'industrie de la céramique méritent de retenir toute notre attention. Il est vrai que ce chapitre contient beaucoup de remarques pratiques destinées aux spécialistes de la prévention des accidents; mais le profane en cette matière en tirera quand même d'utiles enseignements. Les Maladies professionnelles et spécialement la silicose, qui demeure un problème d'une grande actualité, font l'objet d'une étude détaillée. La silicose n'est pas seulement difficile à prévenir; elle pose aussi des problèmes particuliers pour le statisticien et l'actuaire. Enfin, le chapitre L'accident, un processus aléatoire présente surtout un intérêt d'ordre actuariel. La principale question est de savoir comment on peut juger du caractère concluant des expériences sur le risque-accidents. Les explications de ce chapitre ont une importance fondamentale pour la détermination des primes de l'assurance-accidents obligatoire en Suisse. Il vaut donc la peine de se pencher davantage sur ce problème capital, dans lequel se posent des questions intéressantes qui ont reçu une solution dans le cadre de la théorie collective du risque. 


\section{L'appréciation du caractère concluant des expériences en matière de risque-accidents}

En Suisse, l'assurance obligatoire en cas d'accidents est régie par la loi fédérale sur l'assurance en cas de maladie et d'accidents du I3 juin Igrr. Il ressort de cette loi que la prime de chaque entreprise doit être fixée conformément au risque, c'est-à-dire en tenant compte de ses données expérimentales. Dans ce but, on se fonde surtout sur le coût des accidents occasionnés par l'entreprise. Dans ce système, il est nécessaire de pouvoir juger du caractère concluant des expériences. Mais on se heurte à de grandes difficultés, car le processus des accidents est influencé, dans une mesure énorme, par le hasard: le nombre et surtout le coût des accidents sont sujets à des fluctuations importantes. Afin de pouvoir quand même apprécier le caractère concluant des expériences d'un effectif assuré, il faut délimiter le domaine du hasard de ses frais d'accidents. Dans les lignes suivantes, nous allons montrer comment on peut procéder à cette délimitation. Elle se fait sur la base de la distribution des fréquences du coût total des accidents de l'effectif envisagé. Dans ce but, on caractérise l'effectif par $V$ (le nombre des assurés), par $h$ (la fréquence moyenne des accidents d'un assuré) et par $U_{2}$ (la variance de la distribution des frais causés par un accident).

Soient $f(x)$ la distribution des fréquences du nombre des accidents $x$ et $u(z)$ la distribution des fréquences des frais d'accidents $z$ d'un accident $(x, z \geq 0)$. En supposant que ni les accidents, ni les deux variables $x$ et $z$ ne dépendent les uns des autres, la distribution des fréquences des frais d'accidents pour l'effectif assuré est donnée par l'expression

$$
b(z)=\sum_{x=0}^{\infty} f(x)^{(x)} u(z)
$$

où ${ }^{(x)} u(z)$ représente la convolution d'ordre $x$ de $u(z)$. Comme $b(z)$ ne peut pas être tiré directement des observations, il faut l'établir sur la base de $f(x)$ et de $u(z)$.

Dans le domaine des accidents, nous avons affaire à des événements rares dont les probabilités fondamentales sont soumises aux fluctuations du hasard. C'est pourquoi il convient de représenter le processus des accidents par une loi de Poisson composée, avec 
une distribution du type III (Pearson) comme fonction de structure, ce qui nous amène à la distribution binomiale négative

$$
f(x)=\left(\begin{array}{c}
x+p-I \\
x
\end{array}\right)\left(\frac{f_{1}}{p+f_{1}}\right)^{x}\left(\frac{p}{p+f_{1}}\right)^{p}
$$

Dans cette expression, $f_{1}$ désigne la moyenne de $f(x)$ et $p$ la valeur réciproque de la variance de la fonction de structure; une étude a montré qu'on peut poser approximativement $p=\sqrt{V}$. Contrairement à $f(x)$, la fonction $u(z)$ peut être déterminée empiriquement, sous forme de fonction numérique, en dénombrant les accidents d'après leur coût. Elle est extrêmement asymétrique et ne peut guère être représentée par une expression adéquate dont la convolution explicite soit facile à effectuer.

La mise en équation générale de la fonction caractéristique de $b(z)$ nous permet de calculer les moments de $b(z)$ sur la base des moments, numériquement connus, de $f(x)$ et de $u(z)$. Il en résulte, par exemple, comme premier moment de $b(z)$ par rapport à zéro $b_{1}=f_{1}$ (moyenne) et comme deuxième moment de $b(z)$ par rapport à la moyenne $B_{2}=h V .\left(h \sqrt{V}+U_{2}+\mathrm{I}\right)$ (variance). La connaissance de ces caractéristiques statistiques suggère l'idée d'assimiler la distribution des fréquences $b(z)$ à une fonction adéquate et susceptible d'être calculée simplement à l'aide d'une table numérique. La distribution logarithmico-normale à trois paramètres s'y prête spécialement bien; on peut facilement la ramener à la distribution normale ordinaire. Ses trois paramètres peuvent être déterminés de façon que la distribution asymétrique commence au point zéro, qu'elle ait la moyenne $b_{1}$ et qu'elle ait la variance $B_{2}$. En posant $\Delta^{2}=\frac{b_{1}^{2}+B_{2}}{b_{1}{ }^{2}}$, on obtient

$$
b(z)=\frac{\mathrm{I}}{\sqrt{2 \pi}} \frac{\mathrm{I}}{\sqrt{\ln \Delta^{2}} b_{1}}\left(\frac{z \Delta}{b_{1}}\right)^{-\frac{1}{\ln \Delta^{2}} \ln \frac{z \Delta}{b_{1}}-1}
$$

Si nous choisissons une marge de sécurité de 2,5\% de part et d'autre, les intervalles de hasard des frais d'accidents seront les suivants en fonction des paramètres $\left(V, h, U_{2}\right)$ : 


\begin{tabular}{|c|c|c|c|c|c|c|c|}
\hline \multirow{4}{*}{$h$} & \multirow{4}{*}{$V$} & \multicolumn{6}{|c|}{$\mathrm{U}_{2}$} \\
\hline & & \multicolumn{2}{|c|}{ Io } & \multicolumn{2}{|c|}{20} & \multicolumn{2}{|c|}{$4^{\circ}$} \\
\hline & & \multicolumn{6}{|c|}{$\begin{array}{c}\text { Ecart par rapport à la valeur attendue en } \% \\
\text { de cette valeur }\end{array}$} \\
\hline & & en bas & en haut & en bas & en haut & en bas & en haut \\
\hline \multirow{5}{*}{$0, I$} & I0O 000 & 12 & I 3 & I3 & I 5 & I 6 & $x 8$ \\
\hline & 10000 & 25 & $3 I$ & 30 & 39 & 37 & $5 I$ \\
\hline & I 000 & 54 & $9 I$ & 64 & 120 & 75 & I 70 \\
\hline & IOO & 88 & 280 & 93 & 360 & 96 & $44^{\circ}$ \\
\hline & 10 & 99 & 540 & 99 & $57^{\circ}$ & 100 & 580 \\
\hline \multirow{5}{*}{0,4} & 100000 & I I & I 2 & I I & I 2 & 12 & I 3 \\
\hline & IO OOO & 20 & 24 & 22 & 26 & 25 & $3 I$ \\
\hline & I 000 & 39 & 55 & 45 & 68 & 53 & 88 \\
\hline & 100 & 72 & I 60 & 80 & 210 & 87 & 280 \\
\hline & IO & 95 & 410 & 97 & 470 & 99 & 530 \\
\hline \multirow{5}{*}{0,7} & Ioo 000 & I I & 12 & I I & I2 & 12 & 13 \\
\hline & IO OOO & 19 & 23 & 20 & 24 & 22 & 27 \\
\hline & I 000 & 36 & 49 & $4^{\circ}$ & 57 & 46 & 70 \\
\hline & IOO & 65 & 130 & 73 & 160 & $8 \mathrm{I}$ & 220 \\
\hline & Io & 92 & 340 & 96 & 420 & 98 & 490 \\
\hline
\end{tabular}

Prenons, par exemple, un effectif $V$ de Io 000 assurés caractérisé par une fréquence d'accidents $h=0$, I et par une variance de frais $U_{2}=40$. Si cet effectif occasionne des frais d'accidents (normalisés à la moyenne I) de $z=\mathrm{I} 600$, ces frais seront considérés comme s'écartant de façon significative de la valeur attendue $f_{1}=h V=$ I ooo. En effet, la valeur observée se trouve en dehors de l'intervalle $\left(630, x_{5}\right.$ Io) dans lequel tombent les frais dans le 95 pour cent des cas.

Cette méthode permettant de délimiter le domaine du hasard des frais d'accidents peut être utilisée directement pour déterminer les primes. A cet effet, on attribue un poids statistique $g$ aux expériences qui servent à déterminer la prime; on calcule $g$ sur la base des quantités $V, h$ et $U_{2}$ de l'effectif en question. $g$ se situe entre o et I; il est d'autant plus grand que l'intervalle de hasard des frais d'accidents de cet effectif diminue. La quantité $g$ mesure donc le caractère concluant des données expérimentales. Pour fixer la prime, on peut tenir compte des données expérimentales avec un poids $g$ correspondant à leur valeur concluante. Par conséquent, le problème qui consiste à déterminer la prime sur la base des ex- 
périences faites est résolu, du moins théoriquement. Il reste à voir si cette solution est adaptée aux besoins de la pratique et si elle donne des résultats probants.

En résumé, on peut dire que la Caisse nationale suisse d'assurance en cas d'accidents rend un grand service à la science de l'assurance en publiant ses rapports quinquennaux. La conception dont s'inspire son dernier rapport en fait, au point de vue scientifique et documentaire, un instrument précieux qui aide à faire comprendre l'assurance sociale et qui facilite l'étude de son développement. 